\title{
Inclusion theorems of double Deferred Cesàro means II
}

\author{
Richard F. Patterson ${ }^{1}$, Fatih Nuray ${ }^{2} \&$ Metin Başarir $^{3}$ \\ ${ }^{1}$ Department of Mathematics and Statistics, University of North Florida Jacksonville, Florida, 32224 \\ ${ }^{2}$ Department of Mathematical Sciences, Afyon Kocatepe University, Afyonkarahisar, Turkey \\ ${ }^{3}$ Department of Mathematics, Sakarya University, Sakakya, Turkey \\ E-mail: rpatters@unf.edu ${ }^{1}$, fnuray@aku.edu.tr ${ }^{2}$, basarir@sakarya.edu.tr ${ }^{3}$
}

\begin{abstract}
In 1932 R. P. Agnew present a definition for Deferred Cesàro mean. Using this definition R. P. Agnew present inclusion theorems for the deferred and none Deferred Cesàro means. This paper is part 2 of a series of papers that present extensions to the notion of double Deferred Cesàro means. Similar to part 1 this paper uses this definition and the notion of regularity for four dimensional matrices, to present extensions and variations of the inclusion theorems presented by R. P. Agnew in [2].
\end{abstract}

2010 Mathematics Subject Classification. 40B05. 40C05

Keywords. Double Cesàro mean, Deferred Cesàro mean, Double sequence, $R H$-Regular Matrix, $P$-convergent sequences.

\section{Introduction}

This paper is part 2 of a series of papers characterization the inclusion between Cesàro means and double Deferred Cesàro means. In part 1[11] we presented the notion of double Deferred Cesàro means which is a multi-dimensional analog and Agnew's Deferred Cesàro means in [2]. Using this notions and as series of basic results in [11], this paper present a series of inclusion theorems similar to the following: The double Cesáro mean includes $D_{m-1, q_{m}, n-1, p_{n}}$ be a Deferred Cesàro mean with $q_{m}=m, p_{n}=n ; m \neq \alpha_{1}, \alpha_{2}, \ldots$ and $n \neq \beta_{1}, \beta_{2}, \ldots$ with

$$
q_{\alpha_{i}}=\alpha_{i+1}-1 ; i=1,2,3, \ldots, \alpha_{m}
$$

and

$$
p_{\beta_{j}}=\beta_{j+1}-1 ; j+1,2,3, \ldots, \beta_{n}
$$

where $\left\{q_{\alpha_{i}}\right\}$ and $\left\{p_{\beta_{j}}\right\}$ are increasing single dimensional sequences of integers such that $\alpha_{m}>m$ and $\beta_{n}>n$.

\section{Definitions, notations and preliminary results}

The definitions, notations, and preliminary results are similar to those in Part 1 [11] which are restated here for the purpose of completeness.

Definition 2.1 (Pringsheim, 1900). A double sequence $x=\left\{x_{k, l}\right\}$ has a Pringsheim limit $L$ (denoted by $\mathrm{P}-\lim x=L$ ) provided that, given an $\varepsilon>0$ there exists an $N \in \mathbf{N}$ such that $\left|x_{k, l}-L\right|<\varepsilon$ whenever $k, l>N$. Such an $\{x\}$ is described more briefly as "P-convergent". 
Definition 2.2 (Patterson, 2000). A double sequence $\{y\}$ is a double subsequence of $\{x\}$ provided that there exist increasing index sequences $\left\{n_{j}\right\}$ and $\left\{k_{j}\right\}$ such that, if $\left\{x_{j}\right\}=\left\{x_{n_{j}, k_{j}}\right\}$, then $\{\mathrm{y}\}$ is formed by

$\begin{array}{cccc}x_{1} & x_{2} & x_{5} & x_{10} \\ x_{4} & x_{3} & x_{6} & - \\ x_{9} & x_{8} & x_{7} & - \\ - & - & - & -\end{array}$

In [13] Robison presented the following notion of conservative four-dimensional matrix transformation and a Silverman-Toeplitz type characterization of such notion.

Definition 2.3. The four-dimensional matrix $A$ is said to be RH-regular if it maps every bounded $\mathrm{P}$-convergent sequence into a P-convergent sequence with the same P-limit.

The assumption of bounded was added because a double sequence which is P-convergent is not necessarily bounded. Along these same lines, Robison and Hamilton presented a Silverman-Toeplitz type multidimensional characterization of regularity in [3] and [13].

Theorem 2.4. (Hamilton [3], Robison [13]) The four-dimensional matrix $A$ is RH-regular if and only if

$R H_{1}:$ P- $\lim _{m, n} a_{m, n, k, l}=0$ for each $k$ and $l$;

$R H_{2}: \mathrm{P}-\lim _{m, n} \sum_{k, l=0,0}^{\infty, \infty} a_{m, n, k, l}=1$;

$R H_{3}: \mathrm{P}-\lim _{m, n} \sum_{k=0}^{\infty}\left|a_{m, n, k, l}\right|=0$ for each $l$

$R H_{4}: \mathrm{P}-\lim _{m, n} \sum_{l=0}^{\infty}\left|a_{m, n, k, l}\right|=0$ for each $k$;

$R H_{5}: \sum_{k, l=0,0}^{\infty, \infty}\left|a_{m, n, k, l}\right|$ is P-convergent;

$R H_{6}$ : there exist finite positive integers $\Delta$ and $\Gamma$ such that

$\sum_{k, l>\Gamma}\left|a_{m, n, k, l}\right|<\Delta$

The main goals of this paper includes the comparison of double Cesàro mean transformation

$$
(C, 1,1)_{m, n, k, l}:= \begin{cases}\frac{1}{m n}, & \text { if } \quad k \leq m \text { and } l \leq n \\ 0, & \text { if } \quad \text { otherwise }\end{cases}
$$

with the double Deferred Cesàro mean

$$
D_{m, n, k, l}:=\left\{\begin{array}{lll}
\frac{1}{\left(\alpha_{m}-\beta_{m}\right)\left(q_{n}-p_{n}\right)}, & \text { if } \quad \beta_{m}<k \leq \alpha_{m} \text { and } p_{n}<l \leq q_{n}, \\
0, & \text { if } \text { otherwise }
\end{array}\right.
$$

where $\left[p_{n}\right]\left[q_{n}\right]\left[\alpha_{m}\right]$, and $\left[\beta_{m}\right]$ are sequences of nonnegative integers satisfying

$$
\alpha_{m}<\beta_{m} \text {, and } p_{n}<q_{n} \text { for } m, n=1,2 \ldots \text {; }
$$

and

$$
\lim _{m} \beta_{m}=+\infty, \text { and } \lim _{n} q_{n}=+\infty .
$$

Using these four dimensional transformations we shall present a catalog of inclusion theorems such as the following. The four dimensional summability method $M$ include $D_{p_{n}, \alpha_{n}, q_{n}, \beta_{n}}$ where $p_{n}$ and $q_{n}$ for almost all $n$ is a give non-negative integer $p$ if and only if $\alpha_{n}$ and $\beta_{n}$ are almost all positive integers. 


\section{Main results}

Theorem 3.1. The Double Cesàro transformation includes every Double Deferred Cesàro mean of the form $D_{p_{n}, \alpha_{n} q_{n}, \beta_{n}}$ for which $\alpha_{n}$ and $\beta_{n}$ contains almost all positive integers.

Proof. Let $\left[x_{k, l}\right]$ be summable by $D_{p_{n}, \alpha_{n}, q_{n}, \beta_{n}}$ (say to $L$ ) such that $\mathrm{P}-\lim _{m, n} D_{m, n}=L$ and choose two integers $K$ and $L$ large such that $\left[p_{m}\right]$ and $\left[q_{n}\right]$ contains all integers greater than $K$ and $L$, respectively. Thus let $i_{1}=i_{2}=i_{3}=\cdots=i_{K}=1$ and $j_{1}=j_{2}=j_{3}=\cdots=j_{L}=1$ and determine for $m>K$ and $n>L$ index $i_{m}$ and $j_{n}$ is such that $p_{i_{m}}=m$ and $q_{i_{n}}=n$. Since $\lim _{m} i_{m}=+\infty$ and $\lim _{n} j_{n}=+\infty$, it follows

$$
\mathrm{P}-\lim _{m, n} D_{m, n}=L \text { and } \mathrm{P}-\lim _{m, n} D_{i_{m}, j_{n}}=L .
$$

Therefore $[x]$ is summable by $D_{p_{m}, m, q_{n}, n}$ to $L$. The result follows from Lemma 3.3 of [11]. Q.E.D.

Theorem 3.2. The Double Cesàro transformation fails to contain includes $D_{p_{n}, \alpha_{n}, q_{n}, \beta_{n}}$ if there exists an Pringsheim increasing sequence double sequence $\left[\alpha_{k, l}\right]$ of integers whose elements belong to neither $\left[p_{n}\right]$ nor $\left[q_{n}\right]$.

Proof. Let us consider the following

$$
\bar{M}_{m, n}=\left\{\begin{array}{cc}
0, & \text { if } \quad(m, n) \neq\left(\alpha_{m}, \beta_{n}\right) ; m, n=1,2,3, \ldots \\
x_{m, n}, & \text { if } \quad(m, n)=\left(\alpha_{m}, \beta_{n}\right) ; m, n=1,2,3, \ldots
\end{array}\right.
$$

where $[x]$ is a P-divergent double sequence. Let $\left[s_{m, n}\right]$ be double sequence that is mapped by $M$ into $\bar{M}$. Condition $3.2, p_{m} \neq \alpha_{m}$, and $q_{n} \neq \beta_{n}$ asure us that $D_{p_{n}, \alpha_{n}, q_{n}, \beta_{n}}$ sum $[x]$ to zero. Since $M$ fails to sum $[x]$.

Q.E.D.

The following theorem follows from Theorem 3.1 and 3.2 .

Theorem 3.3. The four dimensional summability method $M$ include $D_{p_{n}, \alpha_{n}, q_{n}, \beta_{n}}$ where $p_{n}$ and $q_{n}$ for almost all $n$ is a give non-negative integer $p$ if and only if $\alpha_{n}$ and $\beta_{n}$ are almost all positive integers.

Theorem 3.4. The four dimensional summability method $M$ include $D_{m-1, q_{m}, n-1, \beta_{n}}$ where $q_{m}-m$ and $p_{n}-n$ both increases monotonically with $m$ and $n$, respectively if and only if $q_{m}-m$ and $p_{n}-n$ both are both bounded.

Proof. To establish to sufficiency part not that $q_{m}-m$ and $p_{n}-n$ must have a limit, say $\alpha$ and $\beta$, respectively and that $q_{m}-m=\alpha$ and $p_{n}-n=\beta$ for almost all $m$ and $n$. Thus $\left\{q_{m}\right\}$ and $\left\{p_{n}\right\}$ contains almost all positive integers and Theorem 3.1 grants us the results.

To established the necessary part, suppose $q_{m}-m$ and $p_{n}-n$ increases monotonically with $m$ and $n$ are both unbounded. The goal now is to show that the set of double sequences that are double Cesàro summable are not summable by the double Deferred Cesàro mean. Let $m_{1}=n_{1}=1$ and $m_{2}$ and $n_{2}$ are the smallest integers such that

$$
q_{m}-m>q_{m_{1}}-m_{1} \text { and } p_{n}-n>p_{n_{1}}-n_{1}
$$

Then choose $m_{3}$ and $n_{3}$ to be the smallest integers $m$ and $n$ such that

$$
q_{m}-m>q_{m_{2}}-m_{2} \text { and } p_{n}-n>p_{n_{2}}-n_{2} .
$$


Thus having chosen

$$
m_{1}<m_{2}<\cdots<m_{\alpha} \text { and } n_{1}<n_{2}<\cdots<n_{\beta} .
$$

We then choose $m_{\alpha+1}$ and $n_{\beta+1}$ to be the smallest integers such that

$$
q_{m}-m>q_{m_{\alpha}}-m_{\alpha} \text { and } p_{n}-n>p_{n_{\beta}}-n_{\beta} .
$$

We than define a double sequence $\left\{s_{k, l}\right\}$ as follows:

$$
s_{k, l}=\left\{\begin{array}{ccc}
q_{m_{i}} p_{n_{j}}, & \text { if } \quad k=q_{m_{i}} \text { and } l=p_{n_{j}} ; i, j=1,2,3, \ldots \\
k l, & \text { if } \quad k \neq q_{m_{i}} \text { and } / \text { or } l \neq p_{n_{j}} ; i, j=1,2,3, \ldots
\end{array}\right.
$$

Note $D_{m, n}$ maps $\left\{s_{k, l}\right\}$ into 1 . for all $(m, n)$. Thus $\left\{s_{k, l}\right\}$ is D-summable to 1 . Also $\left\{s_{k, l}\right\}$ is not M-summable, since $\mathrm{P}-\lim _{k, l} \frac{s_{k, l}}{k, l} \neq 0$. Thus the double Cesàro mean is contained in the double Deferred Cesàro mean.

Q.E.D.

Theorem 3.5. Let $D_{m-1, q_{m}, n-1, p_{n}}$ be a Deferred Cesàro mean with $q_{m}=m, p_{n}=n ; m \neq$ $\alpha_{1}, \alpha_{2}, \ldots$ and $n \neq \beta_{1}, \beta_{2}, \ldots$ with

$$
q_{\alpha_{i}}=\alpha_{i+1}-1 ; i=1,2,3, \ldots, \alpha_{m}
$$

and

$$
p_{\beta_{j}}=\beta_{j+1}-1 ; j+1,2,3, \ldots, \beta_{n}
$$

where $\left\{q_{\alpha_{i}}\right\}$ and $\left\{p_{\beta_{j}}\right\}$ are increasing single dimensional sequences of integers such that $\alpha_{m}>m$ and $\beta_{n}>n$. Then $D$ is included in $M$ if and only if $\frac{q_{m}}{m}$ and $\frac{p_{n}}{n}$ are bounded for all $m$ and $n$.

Proof. Note $D_{m-1, m, n-1, n}$ is the identity transformation. Let us consider the ordered pair $(m, n)$ and observe that for each pair $(m, n)$, let

$$
i=i_{m} \text { and } j=j_{n}
$$

be such that $\alpha_{i} \leq m<\alpha_{i+1}$ and $\beta_{j} \leq n<\beta_{j}$. Let $\left\{s_{m, n}\right\}$ be a given double sequence and consider the transformation

$$
M_{m, n}=\frac{1}{m n}\left[\begin{array}{ccccccccc}
s_{1,1} & + & s_{1,2} & + & s_{1,3} & + & \cdots & + & s_{1, n} \\
s_{2,1} & + & s_{2,2} & + & s_{2,3} & + & \cdots & + & s_{2, n} \\
\vdots & \vdots & \vdots & \vdots & \vdots & \vdots & \vdots & \vdots & \vdots \\
s_{m, 1} & + & s_{m, 2} & + & s_{m, 3} & + & \cdots & + & s_{m, n}
\end{array}\right]
$$


Using the definition of double Deferred Cesàro mean we obtain the following

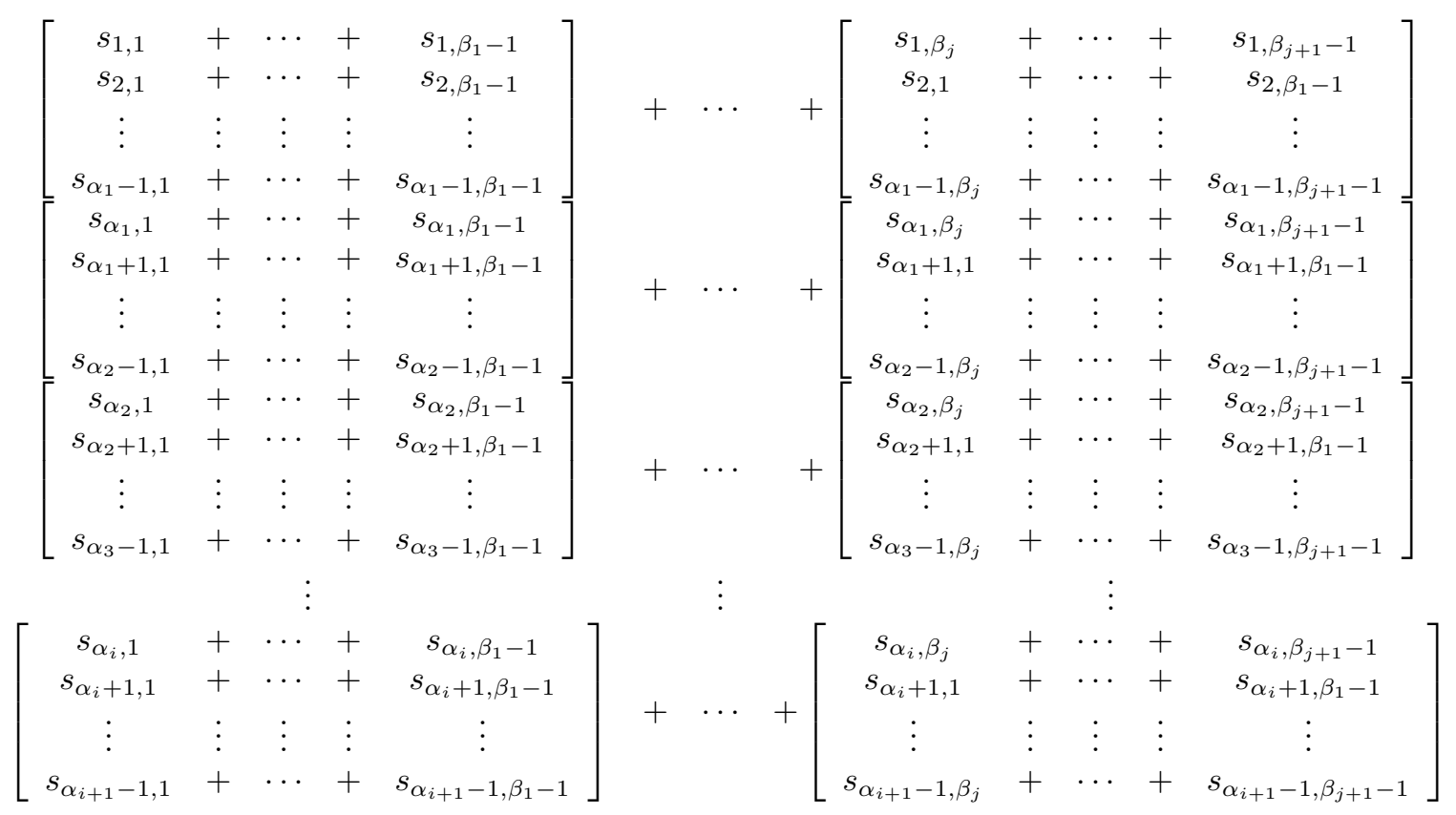

Let us denote the above sum by $\Omega_{m, n}$ and the sum below by $\Lambda_{m, n}$

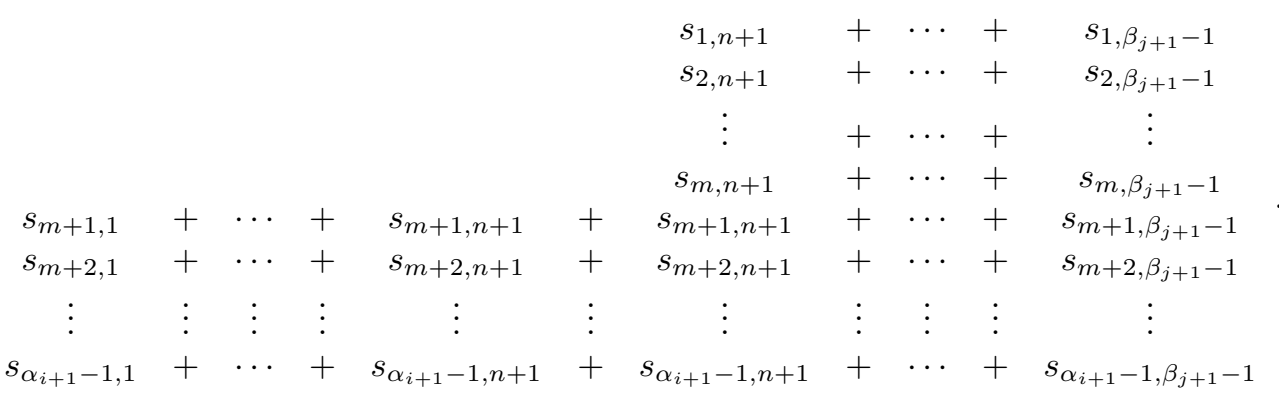

Therefore $M_{m, n}=\frac{1}{m n}\left(\Omega_{m, n}-\Lambda_{m, n}\right)$. It is important to observe that if $m=\alpha_{i+1}-1$ and $/$ or $n=\beta_{j+1}-1$ then the terms in $\Lambda_{m, n}$ will not exist that is if $m=\alpha_{i+1}-1$ and/or $n=\beta_{j+1}-1$ then the terms in the rows and/or columns will not exists. Let us also denote the following sum by 
$\bar{\Omega}_{m, n}$

$$
\begin{array}{ccccccc}
\frac{\sum_{k, l=1,1}^{\alpha_{1}, \beta_{1}} s_{k, l}}{m n} & + & \frac{\alpha_{1}\left(\beta_{2}-\beta_{1}\right)}{m n} D_{0,1} & + & \cdots & + & \frac{\alpha_{1}\left(\beta_{j+1}-\beta_{j}\right)}{m n} D_{0, j} \\
\frac{\left(\alpha_{2}-\alpha_{1}\right) \beta_{1}}{m n} D_{1,0} & + & \frac{\left(\alpha_{2}-\alpha_{1}\right)\left(\beta_{2}-\beta_{1}\right)}{m n} D_{1,1} & + & \cdots & + & \frac{\left(\alpha_{2}-\alpha_{1}\right)\left(\beta_{j+1}-\beta_{j}\right)}{m n} D_{1, j} \\
\frac{\left(\alpha_{3}-\alpha_{2}\right) \beta_{1}}{m n} D_{2,0} & + & \frac{\left(\alpha_{3}-\alpha_{2}\right)\left(\beta_{2}-\beta_{1}\right)}{m n} D_{2,1} & + & \cdots & + & \frac{\left(\alpha_{3}-\alpha_{2}\right)\left(\beta_{j+1}-\beta_{j}\right)}{m n} D_{2, j} \\
+ & + & + & + & + & + & + \\
\vdots & \vdots & \vdots & \vdots & \vdots & \vdots & \vdots \\
+ & + & + & + & + & + & + \\
\frac{\left(\alpha_{i+1}-\alpha_{i}\right) \beta_{1}}{m n} D_{i, 0} & + & \frac{\left(\alpha_{i+1}-\alpha_{i}\right)\left(\beta_{2}-\beta_{1}\right)}{m n} D_{i, 1} & + & \cdots & + & \frac{\left(\alpha_{i+1}-\alpha_{i}\right)\left(\beta_{j+1}-\beta_{j}\right)}{m n} D_{i, j}
\end{array}
$$

and also denote the following sum by $\bar{\Lambda}_{m, n}$

$$
\begin{aligned}
& D_{1, n+1}+\cdots+D_{1, \beta_{j+1}-1} \\
& D_{2, n+1}+\cdots+D_{2, \beta_{j+1}-1}
\end{aligned}
$$

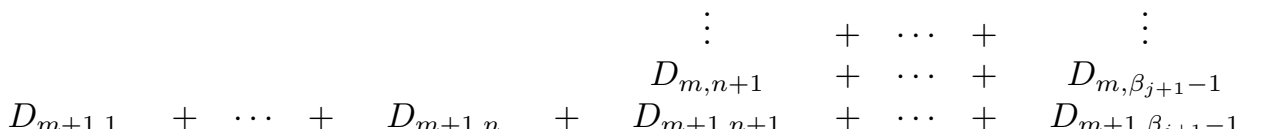

$$
\begin{aligned}
& D_{m+2,1}+\cdots+D_{m+2, n}+D_{m+2, n+1}+\cdots+D_{m+2, \beta_{j+1}-1} \\
& \begin{array}{ccccccccccc}
\vdots & \vdots & \vdots & \vdots & \vdots & \vdots & \vdots & \vdots & \vdots & \vdots & \vdots \\
D_{\alpha_{i+1}-1,1} & + & \cdots & + & D_{\alpha_{i+1}-1, n} & + & D_{\alpha_{i+1}-1, n+1} & + & \cdots & + & D_{\alpha_{i+1}-1, \beta_{j+1}-1}
\end{array}
\end{aligned}
$$

Then we can now rewrite $M_{m, n}$ in the following manner $\bar{\Omega}_{m, n}-\frac{1}{m n} \bar{\Lambda}_{m, n}$. The relation $\bar{\Omega}_{m, n}-$ $\frac{1}{m n} \bar{\Lambda}_{m, n}$ hold for each $(m, n)$ and defines a four-dimensional transformation of the form

$$
\sigma_{m, n}=\sum_{k, l=1,1}^{\infty, \infty} a_{m, n, k, l} s_{k, l}
$$

which carries $D_{m, n}$ into $M_{m, n}$. This transformation clearly satisfies $\mathrm{RH}_{1}$ and $\mathrm{RH}_{2}$. This transformation satisfies $\mathrm{RH}_{3}$ and $\mathrm{RH}_{4}$ only if $\frac{2 \alpha_{i+1}-m-2}{m}$ and $\frac{2 \beta_{j+1}-n-2}{n}$ are bounded respectively for each $(m, n)$, which is equivalent to $\frac{\alpha_{i+1}}{m}$ and $\frac{\alpha_{i+1}^{m}}{m}$ are bounded, which is also equivalent to the boundedness of $\frac{q_{m}}{m}$ and $\frac{p_{n}}{n}$ for each $(m, n)$. Condition $\mathrm{RH}_{5}$ and $\mathrm{RH}_{6}$ hold only when both $\frac{2 \alpha_{i+1}-m-2}{m}$ and $\frac{2 \beta_{j+1}-n-2}{n}$ are bounded, and as above the is equivalent to boundedness of $\frac{q_{m}}{m}$ and $\frac{p_{n}}{n}$ for each $(m, n)$. Since $D$ is a factorable four-dimensional summability matrix the main theorem in [1] assure us that it has an inverse. Thus the result follows for the Robison-Hamilton characterization of regularity.

Q.E.D.

Theorem 3.6. The double Cesáro mean includes $D_{m-1, q_{m}, n-1, p_{n}}$ be a Deferred Cesàro mean with $q_{m}=m, p_{n}=n ; m \neq \alpha_{1}, \alpha_{2}, \ldots$ and $n \neq \beta_{1}, \beta_{2}, \ldots$ with

$$
q_{\alpha_{i}}=\alpha_{i+1}-1 ; i=1,2,3, \ldots, \alpha_{m}
$$

and

$$
p_{\beta_{j}}=\beta_{j+1}-1 ; j+1,2,3, \ldots, \beta_{n}
$$

where $\left\{q_{\alpha_{i}}\right\}$ and $\left\{p_{\beta_{j}}\right\}$ are increasing single dimensional sequences of integers such that $\alpha_{m}>m$ and $\beta_{n}>n$. 
Proof. Observe that for each pair $(m, n)$, let

$$
i=i_{m} \text { and } j=j_{n}
$$

be such that $h_{i} \leq m<h_{i+1}$ and $t_{j} \leq n<t_{j+1}$. Let $\left\{s_{m, n}\right\}$ be a given double sequence and consider the following four dimensional Cesàro transformation

$$
M_{m, n}=\frac{1}{m n}\left[\begin{array}{ccccccccc}
s_{1,1} & + & s_{1,2} & + & s_{1,3} & + & \cdots & + & s_{1, n} \\
s_{2,1} & + & s_{2,2} & + & s_{2,3} & + & \cdots & + & s_{2, n} \\
\vdots & \vdots & \vdots & \vdots & \vdots & \vdots & \vdots & \vdots & \vdots \\
s_{m, 1} & + & s_{m, 2} & + & s_{m, 3} & + & \cdots & + & s_{m, n}
\end{array}\right]
$$

Using the definition of double Deferred Cesàro mean we can rewrite $m n M_{m, n}$ using the following, respectively, $A_{m, n}^{i}, A_{m, n}^{i-1}, A_{m, n}^{i-2} \cdots, A_{m, n}^{\alpha}$ and $K_{m, n}$ where $K_{m, n}$ is

$$
\begin{array}{ccccccc}
s_{1,1} & + & s_{1,2} & + & \cdots & + & s_{1, \beta_{\delta}-1} \\
s_{2,1} & + & s_{2,2} & + & \cdots & + & s_{2, \beta_{\delta}-1} \\
\vdots & + & \vdots & + & \cdots & + & \vdots \\
s_{\beta_{\Delta}-1,1} & + & s_{\beta_{\Delta}-1,2} & + & \cdots & + & s_{\beta_{\Delta}-1, \beta_{\delta}-1}
\end{array}
$$

with $\Delta$ and $\delta$ are 2 or 1 depending on weather $\alpha$ and/ or $\beta$ are odd or even, and the $A$ 's are define below, respectively

$$
\begin{aligned}
& s_{m, n}+s_{m, n-1}+\cdots+s_{m, t_{j}+1} \\
& s_{m-1, n}+s_{m-1, n-1}+\cdots+s_{m-1, t_{j}+1} \\
& \vdots \quad+\quad+\cdots+\quad \vdots \\
& s_{h_{i}+1, n}+s_{h_{i}+1, n-1}+\cdots+s_{h_{i}+1, t_{j}+1}
\end{aligned}
$$

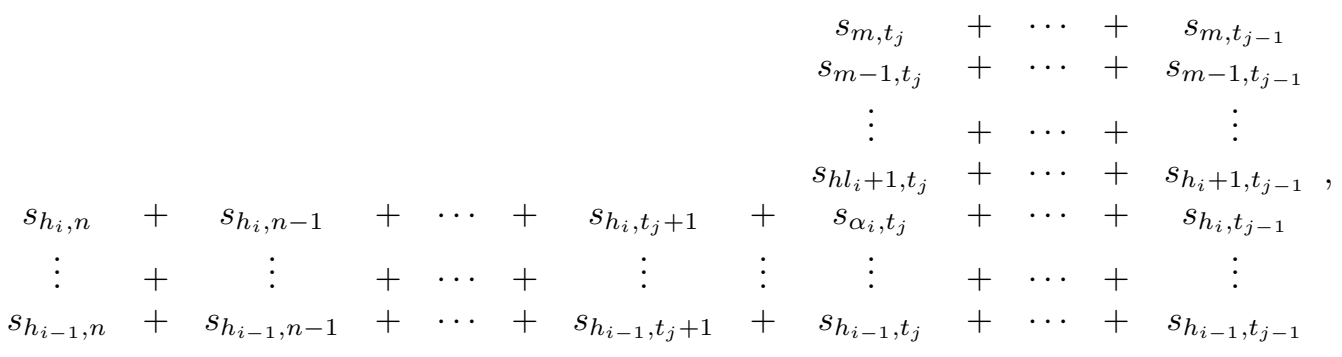

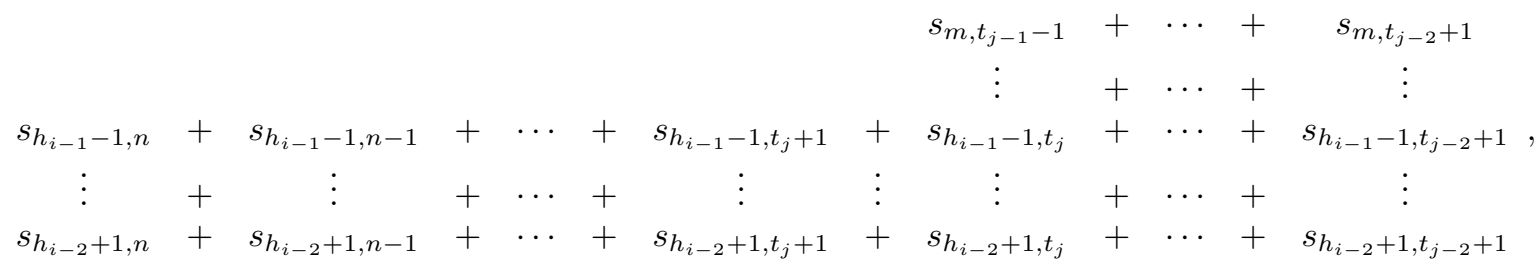




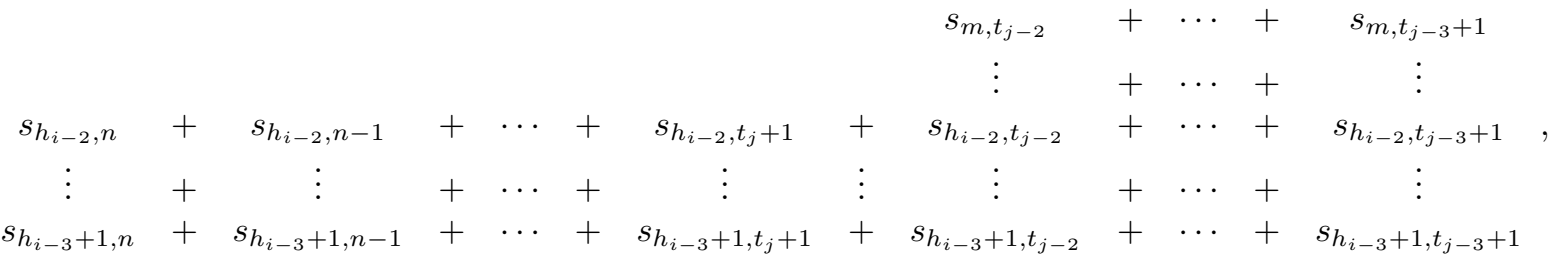

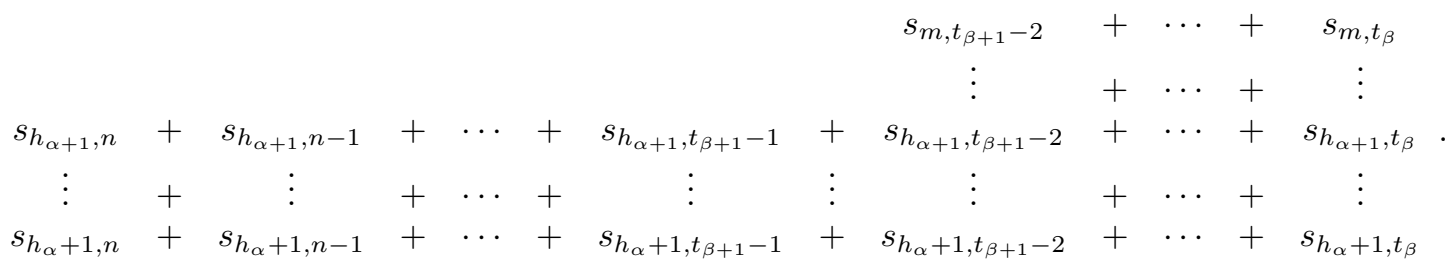

It is clear that

$$
M_{m, n}=\frac{1}{m n}\left[A_{m, n}^{i}+A_{m, n}^{i-1}+A_{m, n}^{i-2}+\cdots+A_{m, n}^{\alpha}+K_{m, n}\right]
$$

Now using the above identities we can rewrite our equation as follow $T_{m, n}=M_{m, n}-\frac{K_{m, n}}{m n}$ and the $D$ 's grants us the following:

$$
\begin{aligned}
& T_{m, n}=\frac{1}{m n}\left[\begin{array}{ccccccc}
D_{m, n} & + & D_{m, n-1} & + & \cdots & + & D_{m, \beta_{j}+1} \\
D_{m-1, n} & + & D_{m-1, n-1} & + & \cdots & + & D_{m-1, \beta_{j}+1} \\
\vdots & \vdots & \vdots & \vdots & \cdots & \vdots & \vdots \\
D_{\alpha_{i}+1, n} & + & D_{\alpha_{i}+1, n-1} & + & \cdots & + & D_{\alpha_{i}+1, \beta_{j}+1}
\end{array}\right]+ \\
& \frac{\left(\alpha_{i}-\alpha_{i-1}+1\right)\left(\beta_{j}+1-n\right)}{m n} D_{\alpha_{i}, n} \quad+\quad \frac{\left(\alpha_{i}-\alpha_{i-1}+1\right)\left(\beta_{j}-\beta_{j-}+1-n\right)}{m n} D_{\alpha_{i-1}, \beta_{j-1}}
\end{aligned}
$$

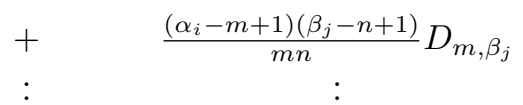

$$
\begin{aligned}
& +\frac{\left(\alpha_{\delta+1}-m+1\right)\left(\beta_{\Delta+1}-\beta_{\Delta}+1\right)}{m n} D_{m, \beta_{\delta}} \quad+\quad \frac{\left(\alpha_{\delta+1}-\alpha_{\delta}+1\right)\left(\beta_{\Delta+1}-\beta_{\Delta}+1\right)}{m n} D_{\alpha_{\delta}, \beta_{\Delta}} \\
& +\quad \frac{\left(\alpha_{\delta+1}-\alpha_{\delta}+1\right)\left(\beta_{\Delta+1}-\beta_{\Delta}+1\right)}{m n} D_{\alpha_{\delta}, n}
\end{aligned}
$$

Since the above equalities define four dimensional RH-regular transformation from $\left\{D_{m, n}\right\}$ to $\left\{T_{m, n}\right\}$ we are granted the if the double sequence $\left\{D_{m, n}\right\}$ convergence to $L$ in the Pringsheim sense then $\left\{T_{m, n}\right\}$ convergence to $L$ in the Pringsheim sense and since the double sequence $\left\{K_{m, n}\right\}$ is bounded then $\left\{M_{m, n}\right\}$ convergence to $L$ in the Pringsheim sense. Thus double Cesàro means includes double Deferred Cesàro means. The completes the proof. 


\section{References}

[1] C. R. Adams, On Summability of Double Series, Trans. Amer. Math. Soc. 34, No.2 (1932), 215-230.

[2] R. P. Agnew, On Deferred Cesàro Means, Annals of Math., 33 (1932), 413-421.

[3] H. J. Hamilton, Transformations of Multiple Sequences, Duke Math. Jour., 2 (1936), 29-60.

[4] H. J. Hamilton, A Generalization of Multiple Sequences Transformation, Duke Math. Jour., 4 (1938), 343-358.

[5] H. J. Hamilton, Change of Dimension in Sequence Transformation, Duke Math. Jour., 4 (1938), 341 - 342.

[6] H. J. Hamilton, Preservation of Partial Limits in Multiple Sequence Transformations, Duke Math. Jour., 5 (1939), 293-297.

[7] G. H. Hardy, Divergent Series. Oxford Univ. Press, London. 1949.

[8] K. Knopp, Zur Theorie der Limitierungsverfahren (Erste Mitteilung), Math. Zeit. 31 (1930), $115-127$.

[9] I. J. Maddox, Some Analogues of Knopp's Core Theorem, Internat. J. Math. \& Math. Sci. 2(4) (1979) 604-614. 2 (1970), 63-65.

[10] R. F. Patterson, Analogues of some Fundamental Theorems of Summability Theory, Internat. J. Math. \& math. Sci. 23(1), (2000), 1-9.

[11] R. F. Patterson \& F. Nuray, Inclusion Theorems of Double Cesáro Means, ( under consideration).

[12] A. Pringsheim, Zur theorie der zweifach unendlichen zahlenfolgen, Mathematische Annalen, 53 (1900) 289-320.

[13] G. M. Robison, Divergent Double Sequences and Series, Amer. Math. Soc. trans. 28 (1926) 50-73. 